\title{
Optimality Conditions and Duality in Nondifferentiable Minimax Fractional Programming with Generalized Convexity ${ }^{1}$
}

\author{
I. AHMAD ${ }^{2}$ AND Z. HUSAIN ${ }^{3}$ \\ Communicated by P. M. Pardalos \\ Published Online: 6 December 2006
}

\begin{abstract}
We establish sufficient optimality conditions for a class of nondifferentiable minimax fractional programming problems involving $(F, \alpha, \rho, d)$ convexity. Subsequently, we apply the optimality conditions to formulate two types of dual problems and prove appropriate duality theorems.
\end{abstract}

Key Words. Minimax fractional programming, sublinear functionals, optimality conditions, duality, generalized convexity.

\section{Introduction}

Schmitendorf (Ref. 1) established necessary and sufficient optimality conditions for a minimax problem. Tanimoto (Ref. 2) applied these optimality conditions to define a dual problem and derived duality theorems, which were extended for the fractional analogue of a generalized minimax problem by Yadav and Mukherjee (Ref. 3). In Ref. 3, Yadav and Mukherjee employed also the optimality conditions of Ref. 1 to construct two dual problems and derived duality theorems for differentiable fractional minimax programming. Chandra and Kumar (Ref. 4) pointed out certain omissions and inconsistencies in the formulation of Yadav and Mukherjee (Ref. 3); they constructed two modified dual problems and proved duality theorems for differentiable fractional minimax programming. Bector and Bhatia (Ref. 5) and Weir (Ref. 6) relaxed the convexity assumptions in the sufficient optimality conditions of Ref. 1, employed the optimality conditions to construct several dual problems which involve pseudoconvex and quasiconvex functions, and discussed weak and strong duality theorems. In Ref. 7, Zalmai

\footnotetext{
${ }^{1}$ The authors thank the referee for valuable suggestions improving the presentation of the paper.

${ }^{2}$ Reader, Department of Mathematics, Aligarh Muslim University, Aligarh, India.

${ }^{3}$ Research Scholar, Department of Mathematics, Aligarh Muslim University, Aligarh, India.
} 
used an infinite-dimensional version of the Gordan theorem of the alternative to derive first-order and second-order necessary optimality conditions for a class of minimax programming problems in a Banach space; he established several sufficient optimality conditions and duality formulations under generalized invexity assumptions. Liu and Wu (Ref. 8) and Ahmad (Ref. 9) recently derived sufficient optimality conditions and duality theorems for minimax fractional programming in the framework of $(F, \rho)$-convex functions and $\rho$-invex functions respectively.

Motivated by various concepts of generalized convexity, Liang et al. (Refs. 10, 11 ) introduced a unified formulation of generalized convexity, which was called $(F, \alpha, \rho, d)$-convexity and obtained some corresponding optimality conditions and duality results for both single-objective fractional problems and multiobjective problems. Recently, Liang and Shi (Ref. 12) obtained sufficient conditions and duality theorems for minimax fractional problem under $(F, \alpha, \rho, d)$-convexity. Lai et al. (Ref. 13) derived necessary and sufficient conditions for nondifferentiable minimax fractional problem with generalized convexity and applied these optimality conditions to construct a parametric dual model and also discussed duality theorems. Lai and Lee (Ref. 14) obtained duality theorems for two parameter-free dual models of nondifferentiable minimax fractional problem involving generalized convexity assumptions. Recently, in view of generalized univexity, Mishra et al. (Ref. 15) extended the results of Refs. 13-14.

In this paper, motivated by Liang et al. (Ref. 10), Lai et al. (Ref. 13), and Lai and Lee (Ref. 14), we establish sufficient conditions for a nondifferentiable minimax fractional programming problem with $(F, \alpha, \rho, d)$-convexity. When the sufficient conditions are utilized, two dual models can be formulated and the usual duality results are presented. In view of the generalized convexity, we extend the results of Refs. 8, 9 and 12-14.

This paper is organized as follows. In Section 2, we give some preliminaries. In Section 3, we establish sufficient optimality conditions. Duality results are presented in Sections 4 and 5.

\section{Notations and Preliminary Results}

Let $R^{n}$ be the $n$-dimensional Euclidean space and let $X$ be an open set in $R^{n}$.

Definition 2.1. A functional $F: X \times X \times R^{n} \rightarrow R$ is said to be sublinear if, $\forall x, \bar{x} \in X$,

(i) $F\left(x, \bar{x} ; a_{1}+a_{2}\right) \leq F\left(x, \bar{x} ; a_{1}\right)+F\left(x, \bar{x} ; a_{2}\right), \quad \forall a_{1}, a_{2} \in R^{n}$,

(ii) $F(x, \bar{x} ; \alpha a)=\alpha F(x, \bar{x} ; a), \quad \forall \alpha \in R_{+}, a \in R^{n}$.

The concept of sublinear functional was given in Ref. 16. By (ii), it is clear that $F(x, \bar{x} ; 0)=0$. 
Based upon the concept of sublinear functional, we recall a unified formulation of generalized convexity [i.e., $(F, \alpha, \rho, d)$-convexity], which was introduced by Liang et al. (Ref. 10) as follows.

Definition 2.2. Let $F: X \times X \times R^{n} \rightarrow R$ be a sublinear functional; let the function $\zeta: X \rightarrow R$ be differentiable at $\bar{x} \in X, \alpha: X \times X \rightarrow R_{+} \backslash\{0\}, \rho \in R$, and $d(.,):. X \times X \rightarrow R$. The function $\zeta$ is said to be $(F, \alpha, \rho, d)$-convex at $\bar{x}$ if

$$
\zeta(x)-\zeta(\bar{x}) \geq F(x, \bar{x} ; \alpha(x, \bar{x}) \nabla \zeta(\bar{x}))+\rho d^{2}(x, \bar{x}), \quad \forall x \in X .
$$

The function $\zeta$ is said to be $(F, \alpha, \rho, d)$-convex over $X$ if it is $(F, \alpha, \rho, d)$-convex at $\bar{x}, \forall x \in X ; \zeta$ is said to be strongly $(F, \alpha, \rho, d)$-convex or $(F, \rho)$-convex if $\rho>0$ or $\rho=0$, respectively.

Special Cases. From Definition 2.2, there are the following special cases.

(I) If $\alpha(x, \bar{x})=1$, for all $x, \bar{x} \in X$, then the $(F, \alpha, \rho, d)$-convexity is the $(F, \rho)$-convexity defined in Ref. 16 .

(II) If $F(x, \bar{x} ; \alpha(x, \bar{x}) \nabla \zeta(\bar{x}))=\nabla \zeta(\bar{x})^{t} \eta(x, \bar{x})$ for a certain map $\eta: X \times$ $X \rightarrow R^{n}$, then the $(F, \alpha, \rho, d)$-convexity is the $\rho$-invexity of Ref. 17 .

(III) If $\rho=0$ or $d(x, \bar{x})=0$ for all $x, \bar{x} \in X$ and if $F(x, \bar{x} ; \alpha(x, \bar{x}) \nabla \zeta(\bar{x}))=$ $\nabla \zeta(\bar{x})^{t} \eta(x, \bar{x})$ for a certain map $\eta: X \times X \rightarrow R^{n}$, then the $(F, \alpha, \rho, d)$ convexity reduces to the invexity introduced in Ref. 18 .

We consider now the following nondifferentiable minimax fractional programming problem:

$$
\begin{aligned}
& \text { (P) } \min _{x \in R^{n}} \sup _{y \in Y} \frac{f(x, y)+\left(x^{t} B x\right)^{1 / 2}}{h(x, y)-\left(x^{t} D x\right)^{1 / 2}}, \\
& \text { s.t. } \quad g(x) \leq 0, \quad x \in X,
\end{aligned}
$$

where $Y$ is a compact subset of $R^{m}, f, h: R^{n} \times R^{m} \rightarrow R$ are $C^{1}$ functions on $R^{n} \times R^{m}$, and $g: R^{n} \rightarrow R^{p}$ is a $C^{1}$ function on $R^{n} ; B$ and $D$ are $n \times n$ positivesemidefinite matrices.

Let

$$
S=\{x \in X: g(x) \leq 0\}
$$

denote the set of all feasible solutions of (P). Any point $x \in S$ is called the feasible point of $(\mathrm{P})$. For each $(x, y) \in R^{n} \times R^{m}$, we define

$$
\phi(x, y)=\frac{f(x, y)+\left(x^{t} B x\right)^{1 / 2}}{h(x, y)-\left(x^{t} D x\right)^{1 / 2}},
$$

such that, for each $(x, y) \in S \times Y$,

$$
f(x, y)+\left(x^{t} B x\right)^{1 / 2} \geq 0 \text { and } h(x, y)-\left(x^{t} D x\right)^{1 / 2}>0 .
$$


For each $x \in S$, we define

$$
J(x)=\left\{j \in J: g_{j}(x)=0\right\},
$$

where

$$
\begin{aligned}
& J=\{1,2, \ldots, p\}, \\
& Y(x)=\left\{y \in Y: \frac{f(x, y)+\left(x^{t} B x\right)^{1 / 2}}{h(x, y)-\left(x^{t} D x\right)^{1 / 2}}=\sup _{z \in Y} \frac{f(x, z)+\left(x^{t} B x\right)^{1 / 2}}{h(x, z)-\left(x^{t} D x\right)^{1 / 2}}\right\} . \\
& K(x)=\left\{(s, t, \tilde{y}) \in N \times R_{+}^{s} \times R^{m s}: 1 \leq s \leq n+1, t=\left(t_{1}, t_{2}, \ldots, t_{s}\right) \in R_{+}^{s},\right. \\
& \text { with } \left.\sum_{i=1}^{s} t_{i}=1, \tilde{y}=\left(\bar{y}_{1}, \bar{y}_{2}, \ldots, \bar{y}_{s}\right), \text { with } \bar{y}_{i} \in Y(x), i=1,2, \ldots, s\right\} .
\end{aligned}
$$

Since $f$ and $h$ are continuously differentiable and $Y$ is compact in $R^{m}$, it follows that, for each $x^{*} \in S, Y\left(x^{*}\right) \neq \emptyset$ and for any $\bar{y}_{i} \in Y\left(x^{*}\right)$, we have a positive constant

$$
k_{\circ}=\phi\left(x^{*}, \bar{y}_{i}\right)=\frac{f\left(x^{*}, \bar{y}_{i}\right)+\left(x^{* t} B x^{*}\right)^{1 / 2}}{h\left(x^{*}, \bar{y}_{i}\right)-\left(x^{* t} D x^{*}\right)^{1 / 2}} .
$$

We shall need the following generalized Schwartz inequality.

Let B be a positive-semidefinite matrix of order $n$. Then, for all $x, w \in R^{n}$,

$$
x^{t} B w \leq\left(x^{t} B x\right)^{1 / 2}\left(w^{t} B w\right)^{1 / 2} .
$$

We observe that equality holds if $B x=\lambda B w$ for some $\lambda \geq 0$. Evidently, if $\left(w^{t} B w\right)^{1 / 2} \leq 1$, we have

$$
x^{t} B w \leq\left(x^{t} B x\right)^{1 / 2} \text {. }
$$

If the functions $f, g, h$ in problem (P) are continuously differentiable with respect to $x \in R^{n}$, then Lai et al. (Ref. 13) derived the following necessary conditions for optimality of (P). In what follows, $\nabla$ stands for the gradient vector with respect to $x$.

Theorem 2.1. Necessary Conditions. If $x^{*}$ is a solution of problem (P) satisfying $x^{* t} B x^{*}>0, x^{* t} D x^{*}>0$ and if $\nabla g_{j}\left(x^{*}\right), j \in J\left(x^{*}\right)$ are linearly independent, then there exist $\left(s, t^{*}, \bar{y}\right) \in K\left(x^{*}\right), k_{\circ} \in R_{+}, w, v \in R^{n}$, and $\mu^{*} \in R_{+}^{p}$ such that

$$
\begin{aligned}
& \sum_{i=1}^{s} t_{i}^{*}\left\{\nabla f\left(x^{*}, \bar{y}_{i}\right)+B w-k_{\circ}\left(\nabla h\left(x^{*}, \bar{y}_{i}\right)-D v\right)\right\}+\nabla \sum_{j=1}^{p} \mu_{j}^{*} g_{j}\left(x^{*}\right)=0, \\
& f\left(x^{*}, \bar{y}_{i}\right)+\left(x^{* t} B x^{*}\right)^{1 / 2}-k_{\circ}\left(h\left(x^{*}, \bar{y}_{i}\right)-\left(x^{* t} D x^{*}\right)^{1 / 2}\right)=0, \quad i=1,2, \ldots, s, \\
& \sum_{j=1}^{p} \mu_{j}^{*} g_{j}\left(x^{*}\right)=0,
\end{aligned}
$$




$$
\begin{aligned}
& t_{i}^{*} \geq 0, \quad i=1,2, \ldots, s, \quad \sum_{i=1}^{s} t_{i}^{*}=1, \\
& w^{t} B w \leq 1, \quad v^{t} D v \leq 1, \\
& \left(x^{* t} B x^{*}\right)^{1 / 2}=x^{* t} B w \\
& \left(x^{* t} D x^{*}\right)^{1 / 2}=x^{* t} D v .
\end{aligned}
$$

Remark 2.1. In the above theorem, both matrices $B$ and $D$ are positive semidefinite. If either $x^{* t} B x^{*}$ or $x^{* t} D x^{*}$ is zero, or if both $B$ and $D$ are singular, then for $\left(s, t^{*}, \bar{y}\right) \in K\left(x^{*}\right)$, we can take a set $Z_{\bar{y}}\left(x^{*}\right)$ as defined in Ref. 13 by

$Z_{\bar{y}}\left(x^{*}\right)=\left\{z \in R^{n}: z^{t} \nabla g_{j}\left(x^{*}\right) \leq 0, j \in J\left(x^{*}\right)\right.$

satisfying one of the following conditions (i), (ii), (iii)\}:

(i)

$$
\begin{aligned}
& x^{* t} B x^{*}>0, x^{* t} D x^{*}=0 \\
& \Rightarrow z^{t}\left(\sum _ { i = 1 } ^ { s } t _ { t } ^ { * } \left\{\nabla f\left(x^{*}, \bar{y}_{i}\right)+B x^{*} /\left(x^{* t} B x^{*}\right)^{1 / 2}\right.\right. \\
& \left.\left.\quad-k_{\circ} \nabla h\left(x^{*}, \bar{y}_{i}\right)\right\}\right)+\left(z^{t}\left(k_{\circ}^{2} D\right) z\right)^{1 / 2}<0,
\end{aligned}
$$

(ii) $\quad x^{* t} B x^{*}=0, x^{* t} D x^{*}>0$

$$
\begin{aligned}
\Rightarrow & z^{t}\left(\sum _ { i = 1 } ^ { s } t _ { i } ^ { * } \left\{\nabla f\left(x^{*}, \bar{y}_{i}\right)-k_{\circ}\left(\nabla h\left(x^{*}, \bar{y}_{i}\right)\right.\right.\right. \\
& \left.\left.\left.-D x^{*} /\left(x^{* t} D x^{*}\right)^{1 / 2}\right)\right\}\right)+\left(z^{t} B z\right)^{1 / 2}<0,
\end{aligned}
$$

(iii) $\quad x^{* t} B x^{*}=0, \quad x^{* t} D x^{*}=0$

$$
\begin{aligned}
\Rightarrow & z^{t}\left(\sum_{i=1}^{s} t_{i}^{*}\left\{\nabla f\left(x^{*}, \bar{y}_{i}\right)-k_{\circ} \nabla h\left(x^{*}, \bar{y}_{i}\right)\right\}\right) \\
& +\left(z^{t}\left(k_{\circ}^{2} D\right) z\right)^{1 / 2}+\left(z^{t} B z\right)^{1 / 2}<0 .
\end{aligned}
$$

If we take $Z_{\bar{y}}\left(x^{*}\right)=\emptyset$ in Theorem 2.1, then the result of Theorem 2.1 still holds.

\section{Sufficient Conditions}

We establish now the sufficient conditions for optimality of $(\mathrm{P})$ under the assumptions of $(F, \alpha, \rho, d)$-convexity. 
Theorem 3.1. Sufficient Conditions. Let $x^{*}$ be a feasible solution of (P) and let there exist a positive integer $s, 1 \leq s \leq n+1, t^{*} \in R_{+}^{s}, \bar{y}_{i} \in Y\left(x^{*}\right), i=$ $1,2, \ldots s, k_{\circ} \in R_{+}, w, v \in R^{n}$, and $\mu^{*} \in R_{+}^{p}$ satisfying relations (2)-(6). If $f\left(., \bar{y}_{i}\right)+(.)^{t} B w$ is $\left(F, \alpha, \rho_{i}, d_{i}\right)$-convex, if $-h\left(., \bar{y}_{i}\right)+(.)^{t} D v$ is $\left(F, \alpha, \bar{\rho}_{i}, \bar{d}_{i}\right)-$ convex, and if $g_{j}($.$) , for j=1,2, \ldots, p$ is $\left(F, \beta_{j}, v_{j}, c_{j}\right)$-convex at $x^{*}$ and

$$
\sum_{i=1}^{s} t_{i}^{*}\left\{\frac{\rho_{i} d_{i}^{2}\left(x, x^{*}\right)}{\alpha\left(x, x^{*}\right)}+k_{\circ} \frac{\bar{\rho}_{i} \bar{d}_{i}^{2}\left(x, x^{*}\right)}{\alpha\left(x, x^{*}\right)}\right\}+\sum_{j=1}^{p} \mu_{j}^{*} v_{j} \frac{c_{j}^{2}\left(x, x^{*}\right)}{\beta_{j}\left(x, x^{*}\right)} \geq 0,
$$

then $x^{*}$ is a global optimal solution of $(\mathrm{P})$.

Proof. Suppose to the contrary that $x^{*}$ is not an optimal solution of (P). Then, there exists an $x \in S$ such that

$$
\sup _{y \in Y} \frac{f(x, y)+\left(x^{t} B x\right)^{1 / 2}}{h(x, y)-\left(x^{t} D x\right)^{1 / 2}}<\sup _{y \in Y} \frac{f\left(x^{*}, y\right)+\left(x^{* t} B x^{*}\right)^{1 / 2}}{h\left(x^{*}, y\right)-\left(x^{* t} D x^{*}\right)^{1 / 2}} .
$$

We note that

$$
\sup _{y \in Y} \frac{f\left(x^{*}, y\right)+\left(x^{* t} B x^{*}\right)^{1 / 2}}{h\left(x^{*}, y\right)-\left(x^{* t} D x^{*}\right)^{1 / 2}}=\frac{f\left(x^{*}, \bar{y}_{i}\right)+\left(x^{* t} B x^{*}\right)^{1 / 2}}{h\left(x^{*}, \bar{y}_{i}\right)-\left(x^{* t} D x^{*}\right)^{1 / 2}}=k_{\circ},
$$

for $\bar{y}_{i} \in Y\left(x^{*}\right), i=1,2, \ldots, s$, and

$$
\frac{f\left(x, \bar{y}_{i}\right)+\left(x^{t} B x\right)^{1 / 2}}{h\left(x, \bar{y}_{i}\right)-\left(x^{t} D x^{*}\right)^{1 / 2}} \leq \sup _{y \in Y} \frac{f(x, y)+\left(x^{t} B x\right)^{1 / 2}}{h(x, y)-\left(x^{t} D x\right)^{1 / 2}} .
$$

Therefore, we have

$$
\frac{f\left(x, \bar{y}_{i}\right)+\left(x^{t} B x\right)^{1 / 2}}{h\left(x, \bar{y}_{i}\right)-\left(x^{t} D x\right)^{1 / 2}}<k_{\circ}, \quad \text { for } i=1,2, \ldots, s .
$$

It follows that

$$
f\left(x, \bar{y}_{i}\right)+\left(x^{t} B x\right)^{1 / 2}-k_{\circ}\left[h\left(x, \bar{y}_{i}\right)-\left(x^{t} D x\right)^{1 / 2}\right]<0, \quad \text { for } i=1,2, \ldots, s .
$$

From relations (1), (3), (5), (6), (8), we obtain

$$
\begin{aligned}
\phi_{\circ}(x) & =\sum_{i=1}^{s} t_{i}^{*}\left\{f\left(x, \bar{y}_{i}\right)+x^{t} B w-k_{\circ}\left(h\left(x, \bar{y}_{i}\right)-x^{t} D v\right)\right\} \\
& \leq \sum_{i=1}^{s} t_{i}^{*}\left\{f\left(x, \bar{y}_{i}\right)+\left(x^{t} B x\right)^{1 / 2}-k_{\circ}\left(h\left(x, \bar{y}_{i}\right)-\left(x^{t} D x\right)^{1 / 2}\right)\right\} \\
<0 & =\sum_{i=1}^{s} t_{i}^{*}\left\{f\left(x^{*}, \bar{y}_{i}\right)+\left(x^{* t} B x^{*}\right)^{1 / 2}-k_{\circ}\left(h\left(x^{*}, \bar{y}_{i}\right)-\left(x^{* t} D x^{*}\right)^{1 / 2}\right)\right\}
\end{aligned}
$$




$$
=\sum_{i=1}^{s} t_{i}^{*}\left\{f\left(x^{*}, \bar{y}_{i}\right)+x^{* t} B w-k_{\mathrm{o}}\left(h\left(x^{*}, \bar{y}_{i}\right)-x^{* t} D v\right)\right\}=\phi_{\circ}\left(x^{*}\right) .
$$

It follows that

$$
\phi_{\circ}(x)<\phi_{\circ}\left(x^{*}\right)
$$

We use the $\left(F, \alpha, \rho_{i}, d_{i}\right)$-convexity of $f\left(., \bar{y}_{i}\right)+(.)^{t} B w$ and the $\left(F, \alpha, \bar{\rho}_{i}, \bar{d}_{i}\right)$ convexity of $-h\left(., \bar{y}_{i}\right)+(.)^{t} D v$ at $x^{*}$ for $i=1,2, \ldots, s$, i.e.,

$$
\begin{aligned}
& f\left(x, \bar{y}_{i}\right)+x^{t} B w-f\left(x^{*}, \bar{y}_{i}\right)-x^{* t} B w \\
& \geq F\left(x, x^{*} ; \alpha\left(x, x^{*}\right)\left(\nabla f\left(x^{*}, \bar{y}_{i}\right)+B w\right)\right)+\rho_{i} d_{i}^{2}\left(x, x^{*}\right), \quad i=1,2, \ldots, s,
\end{aligned}
$$

and

$$
\begin{aligned}
& -h\left(x, \bar{y}_{i}\right)+x^{t} D v+h\left(x^{*}, \bar{y}_{i}\right)-x^{* t} D v \\
& \geq F\left(x, x^{*} ; \alpha\left(x, x^{*}\right)\left(-\nabla h\left(x^{*}, \bar{y}_{i}\right)+D v\right)\right)+\bar{\rho}_{i} \bar{d}_{i}^{2}\left(x, x^{*}\right), \quad i=1,2, \ldots, s .
\end{aligned}
$$

Multiplying (10) by $t_{i}^{*},(11)$ by $t_{i}^{*} k_{\circ}$, and then summing up these inequalities, using the sublinearity of $F$, we have [by (9)]

$$
\begin{aligned}
& F\left(x, x^{*} ; \alpha\left(x, x^{*}\right) \sum_{i=1}^{s} t_{i}^{*}\left\{\nabla f\left(x^{*}, \bar{y}_{i}\right)+B w-k_{\circ}\left(\nabla h\left(x^{*}, \bar{y}_{i}\right)-D v\right)\right\}\right) \\
& +\sum_{i=1}^{s} t_{i}^{*}\left\{\rho_{i} d_{i}^{2}\left(x, x^{*}\right)+k_{\circ} \bar{\rho}_{i} \bar{d}_{i}^{2}\left(x, x^{*}\right)\right\} \leq \phi_{\circ}(x)-\phi_{\circ}\left(x^{*}\right)<0 .
\end{aligned}
$$

Since $\alpha\left(x, x^{*}\right)>0$, by the sublinearity of $F$, we obtain

$$
\begin{aligned}
& F\left(x, x^{*} ; \sum_{i=1}^{s} t_{i}^{*}\left\{\nabla f\left(x^{*}, \bar{y}_{i}\right)+B w-k_{\circ}\left(\nabla h\left(x^{*}, \bar{y}_{i}\right)-D v\right)\right\}\right) \\
& +\sum_{i=1}^{s} t_{i}^{*}\left\{\frac{\rho_{i} d_{i}^{2}\left(x, x^{*}\right)}{\alpha\left(x, x^{*}\right)}+k_{\circ} \frac{\bar{\rho}_{i} \bar{d}_{i}^{2}\left(x, x^{*}\right)}{\alpha\left(x, x^{*}\right)}\right\}<0 .
\end{aligned}
$$

On the other hand, by the $\left(F, \beta_{j}, v_{j}, c_{j}\right)$-convexity of $g_{j}($.$) for j=1,2, \ldots, p$, we have

$$
\begin{aligned}
& g_{j}(x)-g_{j}\left(x^{*}\right) \geq F\left(x, x^{*} ; \beta_{j}\left(x, x^{*}\right) \nabla g_{j}\left(x^{*}\right)\right)+v_{j} c_{j}^{2}\left(x, x^{*}\right), \\
& j=1,2, \ldots, p .
\end{aligned}
$$


By $\mu^{*} \geq 0, \beta\left(x, x^{*}\right)>0$, and the sublinearity of $F$,

$$
\begin{aligned}
& \sum_{j=1}^{p} \mu_{j}^{*} \frac{g_{j}(x)-g_{j}\left(x^{*}\right)}{\beta_{j}\left(x, x^{*}\right)} \\
& \geq F\left(x, x^{*} ; \sum_{j=1}^{p} \mu_{j}^{*} \nabla g_{j}\left(x^{*}\right)\right)+\sum_{j=1}^{p} \mu_{j}^{*} v_{j} \frac{c_{j}^{2}\left(x, x^{*}\right)}{\beta_{j}\left(x, x^{*}\right)}
\end{aligned}
$$

Since the feasibility of $x, \beta\left(x, x^{*}\right)>0$, and (4) imply that

$$
\sum_{j=1}^{p} \mu_{j}^{*} \frac{g_{j}(x)-g_{j}\left(x^{*}\right)}{\beta_{j}\left(x, x^{*}\right)} \leq 0
$$

then (13) leads to

$$
F\left(x, x^{*} ; \sum_{j=1}^{p} \mu_{j}^{*} \nabla g_{j}\left(x^{*}\right)\right)+\sum_{j=1}^{p} \mu_{j}^{*} v_{j} \frac{c_{j}^{2}\left(x, x^{*}\right)}{\beta_{j}\left(x, x^{*}\right)} \leq 0 .
$$

From (2), (12), (14) and the sublinearity of $F$, we have [by (7)]

$$
\begin{aligned}
0= & F\left(x, x^{*} ; \sum_{i=1}^{s} t_{i}^{*}\left\{\nabla f\left(x^{*}, \bar{y}_{i}\right)+B w-k_{\circ}\left(\nabla h\left(x^{*}, \bar{y}_{i}\right)-D v\right)\right\}\right. \\
& \left.+\sum_{j=1}^{p} \mu_{j}^{*} \nabla g_{j}\left(x^{*}\right)\right) \\
\leq & F\left(x, x^{*} ; \sum_{i=1}^{s} t_{i}^{*}\left\{\nabla f\left(x^{*}, \bar{y}_{i}\right)+B w-k_{\circ}\left(\nabla h\left(x^{*}, \bar{y}_{i}\right)-D v\right)\right\}\right) \\
& +F\left(x, x^{*} ; \sum_{j=1}^{p} \mu_{j}^{*} \nabla g_{j}\left(x^{*}\right)\right) \\
< & -\sum_{i=1}^{s} t_{i}^{*}\left\{\frac{\rho_{i} d_{i}^{2}\left(x, x^{*}\right)}{\alpha\left(x, x^{*}\right)}+k_{\circ} \frac{\bar{\rho}_{i} \bar{d}_{i}^{2}\left(x, x^{*}\right)}{\alpha\left(x, x^{*}\right)}\right\}-\sum_{j=1}^{p} \mu_{j}^{*} v_{j} \frac{c_{j}^{2}\left(x, x^{*}\right)}{\beta_{j}\left(x, x^{*}\right)} \\
\leq & 0 .
\end{aligned}
$$

Thus, we have a contradiction. Hence, the proof is complete.

Corollary 3.1. Let $x^{*}$ be a feasible solution of (P) and let there exist a positive integer $s, 1 \leq s \leq n+1, t^{*} \in R_{+}^{s}, \bar{y}_{i} \in Y\left(x^{*}\right), i=1,2, \ldots, s, k_{\circ} \in$ $R_{+}, w, v \in R^{n}$, and $\mu^{*} \in R_{+}^{p}$ satisfying the relations (2)-(6). If $f\left(., \bar{y}_{i}\right)+(.)^{t} B w$ is strongly $\left(F, \alpha, \rho_{i}, d_{i}\right)$-convex or $(F, \alpha)$-convex, if $-h\left(., \bar{y}_{i}\right)+(.)^{t} D v$ is strongly $\left(F, \alpha, \bar{\rho}_{i}, \bar{d}_{i}\right)$-convex or $(F, \alpha)$-convex, and if $g_{j}($.$) , for j=1,2, \ldots, p$, is strongly 
$\left(F, \beta_{j}, v_{j}, c_{j}\right)$-convex or $\left(F, \beta_{j}\right)$-convex at $x^{*}$, then $x^{*}$ is a global optimal solution of $(\mathrm{P})$.

Proof. Under the assumptions of this corollary, we know that inequality, holds [by (7)]. Therefore, $x^{*}$ is a global optimal solution of (P).

\section{Duality Model I}

In this section, we consider the following dual problem to $(\mathrm{P})$ :

(DI) $\max _{(s, t, \bar{y}) \in K(z)} \sup _{(z, \mu, k, v, w) \in H_{1}(s, t, \bar{y})} k$,

where $H_{1}(s, t, \bar{y})$ denotes the set of all $(z, \mu, k, v, w) \in R^{n} \times R_{+}^{p} \times R_{+} \times R^{n} \times R^{n}$ satisfying

$$
\begin{aligned}
& \sum_{i=1}^{s} t_{i}\left\{\nabla f\left(z, \bar{y}_{i}\right)+B w-k\left(\nabla h\left(z, \bar{y}_{i}\right)-D v\right)\right\}+\nabla \sum_{j=1}^{p} \mu_{j} g_{j}(z)=0 \\
& \sum_{i=1}^{s} t_{i}\left\{f\left(z, \bar{y}_{i}\right)+z^{t} B w-k\left(h\left(z, \bar{y}_{i}\right)-z^{t} D v\right)\right\} \geq 0 \\
& \sum_{j=1}^{p} \mu_{j} g_{j}(z) \geq 0 \\
& (s, t, \bar{y}) \in K(z) \\
& w^{t} B w \leq 1, \quad v^{t} D v \leq 1
\end{aligned}
$$

For a triplet $(s, t, \bar{y}) \in K(z)$, if the set $H_{1}(s, t, \bar{y})=\emptyset$, then we define the supremum over it to be $-\infty$. Throughout this section, we denote

$$
\phi_{1}(.)=\sum_{i=1}^{s} t_{i}\left\{f\left(., \bar{y}_{i}\right)+(.)^{t} B w-k\left(h\left(., \bar{y}_{i}\right)-(.)^{t} D v\right)\right\} .
$$

Now, we derive the following weak, strong, and strict converse duality theorems.

Theorem 4.1. Weak Duality. Let $x$ and $(z, \mu, k, v, w, s, t, \bar{y})$ be the feasible solutions of (P) and (DI) respectively. Suppose that $f\left(., \bar{y}_{i}\right)+(.)^{t} B w$ and $-h\left(., \bar{y}_{i}\right)+(.)^{t} D v$, for $i=1,2, \ldots, s$, are respectively $\left(F, \alpha, \rho_{i}, d_{i}\right)$-convex and $\left(F, \alpha, \bar{\rho}_{i}, \bar{d}_{i}\right)$-convex at $z$. Also, let $g_{j}($.$) , for j=1,2, \ldots, p$, be $\left(F, \beta_{j}, v_{j}, c_{j}\right)$ convex at $z$ and let the inequality

$$
\sum_{i=1}^{s} t_{i}\left\{\frac{\rho_{i} d_{i}^{2}(x, z)}{\alpha(x, z)}+k \frac{\bar{\rho}_{i} \bar{d}_{i}^{2}(x, z)}{\alpha(x, z)}\right\}+\sum_{j=1}^{p} \mu_{j} v_{j} \frac{c_{j}^{2}(x, z)}{\beta_{j}(x, z)} \geq 0
$$


hold. Then,

$$
\sup _{y \in Y} \frac{f(x, y)+\left(x^{t} B x\right)^{1 / 2}}{h(x, y)-\left(x^{t} D x\right)^{1 / 2}} \geq k .
$$

Proof. Suppose to the contrary that

$$
\sup _{y \in Y} \frac{f(x, y)+\left(x^{t} B x\right)^{1 / 2}}{h(x, y)-\left(x^{t} D x\right)^{1 / 2}}<k .
$$

Therefore, we get the following relation:

$$
f\left(x, \bar{y}_{i}\right)+\left(x^{t} B x\right)^{1 / 2}-k\left[h\left(x, \bar{y}_{i}\right)-\left(x^{t} D x\right)^{1 / 2}\right]<0, \quad \text { for all } \bar{y}_{i} \in Y .
$$

It follows from $t_{i} \geq 0, i=1,2, \ldots, s$, with $\sum_{i=1}^{s} t_{i}=1$, that

$$
t_{i}\left\{f\left(x, \bar{y}_{i}\right)+\left(x^{t} B x\right)^{1 / 2}-k\left(h\left(x, \bar{y}_{i}\right)-\left(x^{t} D x\right)^{1 / 2}\right)\right\} \leq 0,
$$

with at least one strict inequality because $t=\left(t_{1}, t_{2}, \ldots, t_{s}\right) \neq 0$.

From (1), (16), (19), (21), we have

$$
\begin{aligned}
\phi_{1}(x) & =\sum_{i=1}^{s} t_{i}\left\{f\left(x, \bar{y}_{i}\right)+x^{t} B w-k\left(h\left(x, \bar{y}_{i}\right)-x^{t} D v\right)\right\} \\
& \leq \sum_{i=1}^{s} t_{i}\left\{f\left(x, \bar{y}_{i}\right)+\left(x^{t} B x\right)^{1 / 2}-k\left(h\left(x, \bar{y}_{i}\right)-\left(x^{t} D x\right)^{1 / 2}\right)\right\} \\
& <0 \leq \sum_{i=1}^{s} t_{i}\left\{f\left(z, \bar{y}_{i}\right)+z^{t} B w-k\left(h\left(z, \bar{y}_{i}\right)-z^{t} D v\right)\right\} \\
& =\phi_{1}(z) .
\end{aligned}
$$

Hence,

$$
\phi_{1}(x)<\phi_{1}(z)
$$

Similar to the proof of Theorem 3.1, we have

$$
\begin{aligned}
& F\left(x, z ; \sum_{i=1}^{s} t_{i}\left\{\nabla f\left(z, \bar{y}_{i}\right)+B w-k\left(\nabla h\left(z, \bar{y}_{i}\right)-D v\right)\right\}\right) \\
& +\sum_{i=1}^{s} t_{i}\left\{\frac{\rho_{i} d_{i}^{2}(x, z)}{\alpha(x, z)}+k \frac{\bar{\rho}_{i} \bar{d}_{i}^{2}(x, z)}{\alpha(x, z)}\right\}<0, \\
& \sum_{j=1}^{p} \mu_{j} \frac{g_{j}(x)-g_{j}(z)}{\beta_{j}(x, z)} \geq F\left(x, z ; \sum_{j=1}^{p} \mu_{j} \nabla g_{j}(z)\right)+\sum_{j=1}^{p} \mu_{j} v_{j} \frac{c_{j}^{2}(x, z)}{\beta_{j}(x, z)} .
\end{aligned}
$$


Utilizing the feasibility of $x$ for (P) and (17), we get

$$
\sum_{j=1}^{p} \mu_{j} g_{j}(x) \leq 0 \leq \sum_{j=1}^{p} \mu_{j} g_{j}(z) \text {. }
$$

Therefore, from (23), (24), and $\beta(x, z)>0$, we obtain

$$
F\left(x, z ; \sum_{j=1}^{p} \mu_{j} \nabla g_{j}(z)\right)+\sum_{j=1}^{p} \mu_{j} v_{j} \frac{c_{j}^{2}(x, z)}{\beta_{j}(x, z)} \leq 0 .
$$

Thus, from (15), (22), (25), we get [by (20)]

$$
\begin{aligned}
0= & F\left(x, z ; \sum_{i=1}^{s} t_{i}\left\{\nabla f\left(z, \bar{y}_{i}\right)+B w-k\left(\nabla h\left(z, \bar{y}_{i}\right)-D v\right)\right\}+\sum_{j=1}^{p} \mu_{j} \nabla g_{j}(z)\right) \\
\leq & F\left(x, z ; \sum_{i=1}^{s} t_{i}\left\{\nabla f\left(z, \bar{y}_{i}\right)+B w-k\left(\nabla h\left(z, \bar{y}_{i}\right)-D v\right)\right\}\right) \\
& +F\left(x, z ; \sum_{j=1}^{p} \mu_{j} \nabla g_{j}(z)\right) \\
< & -\sum_{i=1}^{s} t_{i}\left\{\frac{\rho_{i} d_{i}^{2}(x, z)}{\alpha(x, z)}+k \frac{\bar{\rho}_{i} \bar{d}_{i}^{2}(x, z)}{\alpha(x, z)}\right\}-\sum_{j=1}^{p} \mu_{j} v_{j} \frac{c_{j}^{2}(x, z)}{\beta_{j}(x, z)} \\
\leq & 0 .
\end{aligned}
$$

We have a contradiction. Hence, the proof is complete.

Corollary 4.1. Let $x$ and $(z, \mu, k, v, w, s, t, \bar{y})$ be feasible solutions of (P) and (DI) respectively. Suppose that $f\left(., \bar{y}_{i}\right)+(.)^{t} B w$ and $-h\left(., \bar{y}_{i}\right)+(.)^{t} D v$, for $i=1,2, \ldots, s$, are respectively strongly $\left(F, \alpha, \rho_{i}, d_{i}\right)$-convex or $(F, \alpha)$-convex and strongly $\left(F, \alpha, \bar{\rho}_{i}, \bar{d}_{i}\right)$-convex or $(F, \alpha)$-convex at $z$. Also let $g_{j}($.$) , for j=$ $1,2, \ldots, p$, be strongly $\left(F, \beta_{j}, v_{j}, c_{j}\right)$-convex or $\left(F, \beta_{j}\right)$-convex at $z$. Then,

$$
\sup _{y \in Y} \frac{f(x, y)+\left(x^{t} B x\right)^{1 / 2}}{h(x, y)-\left(x^{t} D x\right)^{1 / 2}} \geq k \text {. }
$$

Proof. Under the assumptions of this corollary, we know that the inequality (20) holds. So, we get the corollary from Theorem 4.1.

Theorem 4.2. Strong Duality. Assume that $x^{*}$ is an optimal solution for (P) and let $\nabla g_{j}\left(x^{*}\right), j \in J\left(x^{*}\right)$, be linearly independent. Then, there exist $\left(\bar{s}, \bar{t}, \bar{y}^{*}\right) \in$ $K\left(x^{*}\right)$ and $\left(x^{*}, \bar{\mu}, \bar{k}, \bar{v}, \bar{w}\right) \in H_{1}\left(\bar{s}, \bar{t}, \bar{y}^{*}\right)$ such that $\left(x^{*}, \bar{\mu}, \bar{k}, \bar{v}, \bar{w}, \bar{s}, \bar{t}, \bar{y}^{*}\right)$ is feasible for (DI). Further, if the weak duality holds for all feasible 
$(z, \mu, k, v, w, s, t, \bar{y})$ of (DI), then $\left(x^{*}, \bar{\mu}, \bar{k}, \bar{v}, \bar{w}, \bar{s}, \bar{t}, \bar{y}^{*}\right)$ is optimal for (P) and the two objectives have the same optimal values.

Proof. By Theorem 2.1, there exist $\left(\bar{s}, \bar{t}, \bar{y}^{*}\right) \in K\left(x^{*}\right)$ and $\left(x^{*}, \bar{\mu}, \bar{k}, \bar{v}, \bar{w}\right) \in$ $H_{1}\left(\bar{s}, \bar{t}, \bar{y}^{*}\right)$ such that $\left(x^{*}, \bar{\mu}, \bar{k}, \bar{v}, \bar{w}, \bar{s}, \bar{t}, \bar{y}^{*}\right)$ is feasible for (DI) and

$$
\bar{k}=\frac{f\left(x^{*}, \bar{y}_{i}^{*}\right)+\left(x^{* t} B x^{*}\right)^{1 / 2}}{h\left(x^{*}, \bar{y}_{i}^{*}\right)-\left(x^{* t} D x^{*}\right)^{1 / 2}} .
$$

Since (P) and (DI) have the same objective values, the optimality of this feasible solution follows from Theorem 4.1.

Theorem 4.3. Strict Converse Duality. Let $x^{*}$ and $\left(\bar{z}, \bar{\mu}, \bar{k}, \bar{v}, \bar{w}, \bar{s}, \bar{t}, \bar{y}^{*}\right)$ be the optimal solutions for (P) and (DI), respectively. Suppose that $f\left(., \bar{y}_{i}^{*}\right)+$ $(.)^{t} B \bar{w}$ and $-h\left(., \bar{y}_{i}^{*}\right)+(.)^{t} D \bar{v}$, for $i=1,2, \ldots, \bar{s}$, are respectively $\left(F, \alpha, \rho_{i}, d_{i}\right)$ convex and $\left(F, \alpha, \bar{\rho}_{i}, \bar{d}_{i}\right)$-convex at $\bar{z}$ for all $\left(\bar{s}, \bar{t}, \bar{y}^{*}\right) \in K\left(x^{*}\right)$ and $(\bar{z}, \bar{\mu}, \bar{k}, \bar{v}, \bar{w}) \in$ $H_{1}\left(\bar{s}, \bar{t}, \bar{y}^{*}\right)$. Let $g_{j}($.$) , for j=1,2, \ldots, p$, be $\left(F, \beta_{j}, v_{j}, c_{j}\right)$-convex at $\bar{z}$ and let the inequality

$$
\sum_{i=1}^{\bar{s}} t_{i}\left\{\frac{\rho_{i} d_{i}^{2}\left(x^{*}, \bar{z}\right)}{\alpha\left(x^{*}, \bar{z}\right)}+k \frac{\bar{\rho}_{i} \bar{d}_{i}^{2}\left(x^{*}, \bar{z}\right)}{\alpha\left(x^{*}, \bar{z}\right)}\right\}+\sum_{j=1}^{p} \bar{\mu}_{j} v_{j} \frac{c_{j}^{2}\left(x^{*}, \bar{z}\right)}{\beta_{j}\left(x^{*}, \bar{z}\right)}>0
$$

hold; let $\nabla g_{j}\left(x^{*}\right), j \in J\left(x^{*}\right)$, be linearly independent. Then, $x^{*}=\bar{z}$; that is, $\bar{z}$ is optimal for $(\mathrm{P})$ and

$$
\sup _{y \in Y} \frac{f\left(\bar{z}, \bar{y}^{*}\right)+\left(\bar{z}^{t} B \bar{z}\right)^{1 / 2}}{h\left(\bar{z}, \bar{y}^{*}\right)-\left(\bar{z}^{t} D \bar{z}\right)^{1 / 2}}=\bar{k} .
$$

Proof. We assume that $x^{*} \neq \bar{z}$ and reach a contradiction. From Theorem 4.2, we know that

$$
\sup _{y \in Y} \frac{f\left(x^{*}, \bar{y}^{*}\right)+\left(x^{* t} B x^{*}\right)^{1 / 2}}{h\left(x^{*}, \bar{y}^{*}\right)-\left(x^{* t} D x^{*}\right)^{1 / 2}}=\bar{k} .
$$

Similar to the proof of Theorem 4.1, we have

$$
\begin{aligned}
\frac{\phi_{1}\left(x^{*}\right)-\phi_{1}(\bar{z})}{\alpha\left(x^{*}, \bar{z}\right)} & \geq F\left(x^{*}, \bar{z} ; \sum_{i=1}^{\bar{s}} \bar{t}_{i}\left\{\nabla f\left(\bar{z}, \bar{y}_{i}^{*}\right)+B \bar{w}-\bar{k}\left(\nabla h\left(\bar{z}, \bar{y}_{i}^{*}\right)-D \bar{v}\right)\right\}\right) \\
& +\sum_{i=1}^{\bar{s}} \bar{t}_{i}\left\{\frac{\rho_{i} d_{i}^{2}\left(x^{*}, \bar{z}\right)}{\alpha\left(x^{*}, \bar{z}\right)}+\bar{k} \frac{\bar{\rho}_{i} \bar{d}_{i}^{2}\left(x^{*}, \bar{z}\right)}{\alpha\left(x^{*}, \bar{z}\right)}\right\}
\end{aligned}
$$




$$
\sum_{j=1}^{p} \bar{\mu}_{j} \frac{g_{j}\left(x^{*}\right)-g_{j}(\bar{z})}{\beta_{j}\left(x^{*}, \bar{z}\right)} \geq F\left(x^{*}, \bar{z} ; \sum_{j=1}^{p} \bar{\mu}_{j} \nabla g_{j}(\bar{z})\right)+\sum_{j=1}^{p} \bar{\mu}_{j} v_{j} \frac{c_{j}^{2}\left(x^{*}, \bar{z}\right)}{\beta_{j}\left(x^{*}, \bar{z}\right)}
$$

By both the feasibility of $x^{*}$ and (17), we have

$$
\sum_{j=1}^{p} \bar{\mu}_{j} g_{j}\left(x^{*}\right) \leq 0 \leq \sum_{j=1}^{p} \bar{\mu}_{j} g_{j}(\bar{z})
$$

Thus, from (28) and $\beta\left(x^{*}, \bar{z}\right)>0$, we obtain

$$
F\left(x^{*}, \bar{z} ; \sum_{j=1}^{p} \bar{\mu}_{j} \nabla g_{j}(\bar{z})\right)+\sum_{j=1}^{p} \bar{\mu}_{j} v_{j} \frac{c_{j}^{2}\left(x^{*}, \bar{z}\right)}{\beta_{j}\left(x^{*}, \bar{z}\right)} \leq 0 .
$$

Relations (15), (26), (29) along with the sublinearity of $F$ yield

$$
\begin{aligned}
& F\left(x^{*}, \bar{z} ; \sum_{i=1}^{\bar{s}} \bar{t}_{i}\left\{\nabla f\left(\bar{z}, \bar{y}_{i}^{*}\right)+B \bar{w}-\bar{k}\left(\nabla h\left(\bar{z}, \bar{y}_{i}^{*}\right)-D \bar{v}\right)\right\}\right) \\
& \geq-F\left(x^{*}, \bar{z} ; \sum_{j=1}^{p} \bar{\mu}_{j} \nabla g_{j}(\bar{z})\right) \\
& \geq \sum_{j=1}^{p} \bar{\mu}_{j} v_{j} \frac{c_{j}^{2}\left(x^{*}, \bar{z}\right)}{\beta_{j}\left(x^{*}, \bar{z}\right)} \\
& >-\sum_{i=1}^{\bar{s}} \bar{t}_{i}\left\{\frac{\rho_{i} d_{i}^{2}\left(x^{*}, \bar{z}\right)}{\alpha\left(x^{*}, \bar{z}\right)}+\bar{k} \frac{\bar{\rho}_{i} \bar{d}_{i}^{2}\left(x^{*}, \bar{z}\right)}{\alpha\left(x^{*}, \bar{z}\right)}\right\} .
\end{aligned}
$$

Hence, from (27), (30), and $\alpha\left(x^{*}, \bar{z}\right)>0$, we get

$$
\phi_{1}\left(x^{*}\right)-\phi_{1}(\bar{z})>0 .
$$

Now, we get the following relation [by (16)]:

$$
\begin{aligned}
& \sum_{i=1}^{\bar{s}} \bar{t}_{i}\left\{f\left(x^{*}, \bar{y}_{i}^{*}\right)+\left(x^{* t} B x^{*}\right)^{1 / 2}-\bar{k}\left(h\left(x^{*}, \bar{y}_{i}^{*}\right)-\left(x^{* t} D x^{*}\right)^{1 / 2}\right)\right\} \\
& >\sum_{i=1}^{\bar{s}} \bar{t}_{i}\left\{f\left(\bar{z}, \bar{y}_{i}^{*}\right)+\left(\bar{z}^{t} B \bar{z}\right)^{1 / 2}-\bar{k}\left(h\left(\bar{z}, \bar{y}_{i}^{*}\right)-\left(\bar{z}^{t} D \bar{z}\right)^{1 / 2}\right)\right\} \geq 0 .
\end{aligned}
$$

Therefore, there exists a certain $i_{\circ}$, such that

$$
f\left(x^{*}, \bar{y}_{i_{\circ}}^{*}\right)+\left(x^{* t} B x^{*}\right)^{1 / 2}-\bar{k}\left[h\left(x^{*}, \bar{y}_{i_{\circ}}^{*}\right)-\left(x^{* t} D x^{*}\right)^{1 / 2}\right]>0 .
$$


It follows that

$$
\sup _{y \in Y} \frac{f\left(x^{*}, \bar{y}^{*}\right)+\left(x^{* t} B x^{*}\right)^{1 / 2}}{f\left(x^{*}, \bar{y}^{*}\right)-\left(x^{* t} D x^{*}\right)^{1 / 2}} \geq \frac{f\left(x^{*}, \bar{y}_{i_{\circ}}^{*}\right)+\left(x^{* t} B x^{*}\right)^{1 / 2}}{h\left(x^{*}, \bar{y}_{i_{\circ}}^{*}\right)-\left(x^{* t} D x^{*}\right)^{1 / 2}}>\bar{k} .
$$

Finally, we have a contradiction and the proof is complete.

Remark 4.1. If we take

$F(x, \bar{x} ; \alpha(x, \bar{x}) \nabla f(\bar{x}))=(x-\bar{x})^{t} \nabla f(\bar{x}), \quad$ for $x, \bar{x} \in X$,

in Theorems 4.1-4.3, we get Theorems 4.1-4.3 in Ref. 13. If we remove the quadratic terms from the numerator and denominator of the objective function and if take $\alpha(x, \bar{x})=1$, for all $x \in X$, we obtain Theorems 4.1-4.3 in Ref. 8. Also, if we take

$$
F(x, \bar{x} ; \alpha(x, \bar{x}) \nabla f(\bar{x}))=\eta(x, \bar{x})^{t} \nabla f(\bar{x})
$$

for a certain map $\eta: X \times X \rightarrow R^{n}$ and remove the quadratic terms from the numerator and denominator of the objective function, we get Theorems 4.1-4.3 in Ref. 9.

\section{Duality Model II}

In this section, we introduce a Wolfe type dual model to problem $(\mathrm{P})$. In order to discuss the following duality model, we state first another version of Theorem 2.1, by replacing the parameter $k_{\circ}$ with $\frac{f\left(\bar{x}, \bar{y}_{i}\right)+\left(\bar{x}^{t} B \bar{x}\right)^{1 / 2}}{h\left(\bar{x}, \bar{y}_{i}\right)-\left(\bar{x}^{t} D \bar{x}\right)^{1 / 2}}$ and by rewriting the multiplier functions associated with the inequality constraints.

Theorem 5.1. Let $\bar{x}$ be a solution for (P) and let $\nabla g_{j}(\bar{x}), j \in J(\bar{x})$ be linearly independent. Then, there exist $(\bar{s}, \bar{t}, \bar{y}) \in K(\bar{x})$ and $\bar{\mu} \in R_{+}^{p}$ such that

$$
\begin{aligned}
& \sum_{i=1}^{\bar{s}} \bar{t}_{i}\left\{\left(h\left(\bar{x}, \bar{y}_{i}\right)-\left(\bar{x}^{t} D \bar{x}\right)^{1 / 2}\right)\left(\nabla f\left(\bar{x}, \bar{y}_{i}\right)+B w\right)\right. \\
& \left.\quad-\left(f\left(\bar{x}, \bar{y}_{i}\right)+\left(\bar{x}^{t} B \bar{x}\right)^{1 / 2}\right)\left(\nabla h\left(\bar{x}, \bar{y}_{i}\right)-D v\right)\right\}+\sum_{j=1}^{p} \bar{\mu}_{j} \nabla g_{j}(\bar{x})=0, \\
& \sum_{j=1}^{p} \bar{\mu}_{j} g_{j}(\bar{x}) \geq 0, \\
& \bar{\mu} \in R_{+}^{p}, \quad \bar{t}_{i} \geq 0, \quad \sum_{i=1}^{\bar{s}} \bar{t}_{i}=1, \quad \bar{y}_{i} \in Y(\bar{x}), \quad i=1,2, \ldots, \bar{s} . \\
& \text { (DII) } \max _{(x, t, \bar{y}) \in K(z)} \sup _{(z, \mu, v, w) \in H_{2}(s, t, \bar{y})} F(z),
\end{aligned}
$$


where

$$
F(z)=\sup _{y \in Y} \frac{f(z, y)+\left(z^{t} B x\right)^{1 / 2}}{h(z, y)-\left(z^{t} D x\right)^{1 / 2}}
$$

and $H_{2}(s, t, \bar{y})$ denotes the set of all $(z, \mu, v, w) \in R^{n} \times R_{+}^{p} \times R^{n} \times R^{n}$ satisfying

$$
\begin{aligned}
& \sum_{i=1}^{s} t_{i}\left\{\left(h\left(z, \bar{y}_{i}\right)-\left(z^{t} D z\right)^{1 / 2}\right)\left(\nabla f\left(z, \bar{y}_{i}\right)+B w\right)\right. \\
& \left.\quad-\left(f\left(z, \bar{y}_{i}\right)+\left(z^{t} B z\right)^{1 / 2}\right)\left(\nabla h\left(z, \bar{y}_{i}\right)-D v\right)\right\}+\sum_{j=1}^{p} \bar{\mu}_{j} \nabla g_{j}(z)=0, \\
& \sum_{j=1}^{p} \mu_{j} g_{j}(z) \geq 0, \\
& (s, t, \bar{y}) \in K(z), \\
& \left(z^{t} B z\right)^{1 / 2}=z^{t} B w, \quad\left(z^{t} D z\right)^{1 / 2}=z^{t} D v, \quad w^{t} B w \leq 1, \quad v^{t} D v \leq 1 .
\end{aligned}
$$

For a triplet $(s, t, \bar{y}) \in K(z)$, if the set $H_{2}(s, t, \bar{y})$ is empty, then we define the supremum over it to be $-\infty$. For convenience, we let

$$
\begin{array}{r}
\phi_{2}(.)=\sum_{i=1}^{s} t_{i}\left\{\left(h\left(z, \bar{y}_{i}\right)-z^{t} D v\right)\left(f\left(., \bar{y}_{i}\right)+(.)^{t} B w\right)\right. \\
\left.-\left(f\left(z, \bar{y}_{i}\right)+z^{t} B w\right)\left(h\left(., \bar{y}_{i}\right)-(.)^{t} D v\right)\right\} .
\end{array}
$$

Then, we can establish the following weak, strong, and strict converse duality theorems.

Theorem 5.2. Weak Duality. Let $x$ and $(z, \mu, v, w, s, t, \bar{y})$ be the feasible solutions of (P) and (DII) respectively. Suppose that $f\left(., \bar{y}_{i}\right)+(.)^{t} B w$ and $-h\left(., \bar{y}_{i}\right)+(.)^{t} D v$, for $i=1,2, \ldots, s$, are respectively $\left(F, \alpha, \rho_{i}, d_{i}\right)$-convex and $\left(F, \alpha, \bar{\rho}_{i}, \bar{d}_{i}\right)$-convex at $z$. Also, let $g_{j}($.$) , for j=1,2, \ldots, p$, be $\left(F, \beta_{j}, v_{j}, c_{j}\right)$ convex at $z$ and let the inequality

$$
\begin{aligned}
& \sum_{i=1}^{s} t_{i}\left\{\frac{\left(h\left(z, \bar{y}_{i}\right)-\left(z^{t} D z\right)^{1 / 2}\right) \rho_{i} d_{i}^{2}(x, z)}{\alpha(x, z)}+\frac{\left(f\left(z, \bar{y}_{i}\right)+\left(z^{t} B z\right)^{1 / 2}\right) \bar{\rho}_{i} \bar{d}_{i}^{2}(x, z)}{\alpha(x, z)}\right\} \\
& +\sum_{j=1}^{p} \mu_{j} v_{j} \frac{c_{j}^{2}(x, z)}{\beta_{j}(x, z)} \geq 0
\end{aligned}
$$

hold. Then,

$$
\sup _{y \in Y} \frac{f(x, y)+\left(x^{t} B x\right)^{1 / 2}}{h(x, y)-\left(x^{t} D x\right)^{1 / 2}} \geq F(z) .
$$


Proof. Suppose to the contrary that

$$
\sup _{y \in Y} \frac{f(x, y)+\left(x^{t} B x\right)^{1 / 2}}{h(x, y)-\left(x^{t} D x\right)^{1 / 2}}<F(z) .
$$

Since $\bar{y}_{i} \in Y(z), i=1,2, \ldots, s$, we get

$$
F(z)=\frac{f\left(z, \bar{y}_{i}\right)+\left(z^{t} B z\right)^{1 / 2}}{h\left(z, \bar{y}_{i}\right)-\left(z^{t} D z\right)^{1 / 2}} .
$$

By (37) and (38), we have

$$
\begin{aligned}
& {\left[h\left(z, \bar{y}_{i}\right)-\left(z^{t} D z\right)^{1 / 2}\right]\left[f\left(x, \bar{y}_{i}\right)-\left(x^{t} B x\right)^{1 / 2}\right]} \\
& -\left[f\left(z, \bar{y}_{i}\right)+\left(z^{t} B z\right)^{1 / 2}\right]\left[h\left(x, \bar{y}_{i}\right)-\left(x^{t} D x\right)^{1 / 2}\right]<0,
\end{aligned}
$$

for all $i=1,2, \ldots, s$ and $\bar{y}_{i} \in Y$. From $\bar{y}_{i} \in Y(z) \subset Y$ and $t \in R_{+}^{s}$, with $\sum_{i=1}^{s} t_{i}=$ 1 , we obtain

$$
\begin{aligned}
\sum_{i=1}^{s} t_{i} & \left\{\left[h\left(z, \bar{y}_{i}\right)-\left(z^{t} D z\right)^{1 / 2}\right]\left[f\left(x, \bar{y}_{i}\right)+\left(x^{t} B x\right)^{1 / 2}\right]\right. \\
& \left.-\left[f\left(z, \bar{y}_{i}\right)+\left(z^{t} B z\right)^{1 / 2}\right]\left[h\left(x, \bar{y}_{i}\right)-\left(x^{t} D x\right)^{1 / 2}\right]\right\}<0 .
\end{aligned}
$$

By (1), (35), and (39), we have

$$
\begin{aligned}
\phi_{2}(x)= & \sum_{i=1}^{s} t_{i}\left\{\left(h\left(z, \bar{y}_{i}\right)-z^{t} D v\right)\left(f\left(x, \bar{y}_{i}\right)+x^{t} B w\right)\right. \\
& \left.-\left(f\left(z, \bar{y}_{i}\right)+z^{t} B w\right)\left(h\left(x, \bar{y}_{i}\right)-x^{t} D v\right)\right\} \\
\leq & \sum_{i=1}^{s} t_{i}\left\{\left(h\left(z, \bar{y}_{i}\right)-\left(z^{t} D z\right)^{1 / 2}\right)\left(f\left(x, \bar{y}_{i}\right)+\left(x^{t} B x\right)^{1 / 2}\right)\right. \\
& \left.\left.-\left(f\left(z, \bar{y}_{i}\right)+z^{t} B z\right)^{1 / 2}\right)\left(h\left(x, \bar{y}_{i}\right)-\left(x^{t} D x\right)^{1 / 2}\right)\right\} \\
<0= & \phi_{2}(z) .
\end{aligned}
$$

Hence,

$$
\phi_{2}(x)<\phi_{2}(z)
$$

Using the $\left(F, \alpha, \rho_{i}, d_{i}\right)$-convexity of $f\left(., \bar{y}_{i}\right)+(.)^{t} B w$ and the $\left(F, \alpha, \bar{\rho}_{i}, \bar{d}_{i}\right)$ convexity of $-h\left(., \bar{y}_{i}\right)+(.)^{t} D v$ at $z$ for $i=1,2, \ldots, s$, i.e.,

$$
\begin{aligned}
& f\left(x, \bar{y}_{i}\right)+x^{t} B w-f\left(z, \bar{y}_{i}\right)-z^{t} B w \\
& \geq F\left(x, z ; \alpha(x, z)\left(\nabla f\left(z, \bar{y}_{i}\right)+B w\right)\right)+\rho_{i} d_{i}^{2}(x, z), \quad i=1,2, \ldots, s, \\
& -h\left(x, \bar{y}_{i}\right)+x^{t} D v+h\left(z, \bar{y}_{i}\right)-z^{t} D v \\
& \geq F\left(x, z ; \alpha(x, z)\left(-\Delta h\left(z, \bar{y}_{i}\right)+D v\right)\right)+\bar{\rho}_{i} \bar{d}_{i}^{2}(x, z), \quad i=1,2, \ldots, s .
\end{aligned}
$$


Multiplying (41) by $t_{i}\left[h\left(z, \bar{y}_{i}\right)-\left(z^{t} D z\right)^{1 / 2}\right],(42)$ by $t_{i}\left[f\left(z, \bar{y}_{i}\right)+\left(z^{t} B z\right)^{1 / 2}\right]$, and then summing up these inequalities with the sublinearity of $F$, we get

$$
\begin{aligned}
\frac{\phi_{2}(x)-\phi_{2}(z)}{\alpha(z, x)} \geq F\left(x, z ; \sum_{i=1}^{s} t_{i}\left\{\left(h\left(z, \bar{y}_{i}\right)-\left(z^{t} D z\right)^{1 / 2}\right)\left(\nabla f\left(z, \bar{y}_{i}\right)+B w\right)\right.\right. \\
\left.\left.-\left(f\left(z, \bar{y}_{i}\right)+\left(z^{t} B z\right)^{1 / 2}\right)\left(\nabla h\left(z, \bar{y}_{i}\right)-D v\right)\right\}\right) \\
+\sum_{i=1}^{s} t_{i}\left\{\frac{\left(h\left(z, \bar{y}_{i}\right)-\left(z^{t} D z\right)^{1 / 2}\right) \rho_{i} d_{i}^{2}(x, z)}{\alpha(x, z)}\right. \\
\left.+\frac{\left(f\left(z, \bar{y}_{i}\right)+\left(z^{t} B z\right)^{1 / 2}\right) \bar{\rho}_{i} \bar{d}_{i}^{2}(x, z)}{\alpha(x, z)}\right\},
\end{aligned}
$$

which by using the $\left(F, \beta_{j}, v_{j}, c_{j}\right)$-convexity of $g_{j}(),.(32)$, and (36), along with the sublinearity of $F$, yields

$$
\begin{aligned}
\frac{\phi_{2}(x)-\phi_{2}(z)}{\alpha(z, x)} & \geq F\left(x, z ;-\sum_{j=1}^{p} \mu_{j} \nabla g_{j}(z)\right)-\sum_{j=1}^{p} \mu_{j} v_{j} \frac{c_{j}^{2}(x, z)}{\beta_{j}(x, z)} \\
& \geq \frac{-\sum_{j=1}^{p} \mu_{j} g_{j}(x)-\sum_{j=1}^{p} \mu_{j} g_{j}(z)}{\beta_{j}(x, z)} \\
& \geq 0,
\end{aligned}
$$

by the feasibility of $x,(33)$, and $\beta_{j}(x, z)>0$. Since $\alpha(x, z)>0$, we get $\phi_{2}(x) \geq$ $\phi_{2}(z)$, which contradicts (40). Hence, the proof is complete.

Corollary 5.1. Let $x$ and $(z, \mu, v, w, s, t, \bar{y})$ be feasible solutions of (P) and (DII) respectively. Let $f\left(., \bar{y}_{i}\right)+(.)^{t} B w$ and $-h\left(., \bar{y}_{i}\right)+(.)^{t} D v$, for $i=1,2, \ldots, s$, be respectively strongly $\left(F, \alpha, \rho_{i}, d_{i}\right)$-convex or $(F, \alpha)$-convex and strongly $\left(F, \alpha, \bar{\rho}_{i}, \bar{d}_{i}\right)$-convex or $(F, \alpha)$-convex at $z$. Also, let $g_{j}($.$) , for$ $j=1,2, \ldots, p$, be strongly $\left(F, \beta_{j}, v_{j}, c_{j}\right)$-convex or $\left(F, \beta_{j}\right)$-convex at $z$. Then,

$$
\sup _{y \in Y} \frac{f(x, y)+\left(x^{t} B x\right)^{1 / 2}}{h(x, y)-\left(x^{t} D x\right)^{1 / 2}} \geq F(z) \text {. }
$$

Proof. Under the assumptions of this corollary, we know that the inequality (36) holds. So, we can get the corollary from Theorem 5.2.

Theorem 5.3. Strong Duality. Let $x^{*}$ be an optimal solution for $(\mathrm{P})$ and let $\nabla g_{j}\left(x^{*}\right), j \in J\left(x^{*}\right)$, be linearly independent. Then, there exist $\left(\bar{s}, \bar{t}, \bar{y}^{*}\right) \in$ $K\left(x^{*}\right)$ and $\left(x^{*}, \bar{\mu}, \bar{v}, \bar{w}\right) \in H_{2}\left(\bar{s}, \bar{t}, \bar{y}^{*}\right)$ such that $\left(x^{*}, \bar{\mu}, \bar{v}, \bar{w}, \bar{s}, \bar{t}, \bar{y}^{*}\right)$ is feasible for (DII). Further, let the weak duality holds for all feasible $(z, \mu, v, w, s, t, \bar{y})$ of 
(DII). Then, ( $\left.x^{*}, \bar{\mu}, \bar{v}, \bar{w}, \bar{s}, \bar{t}, \bar{y}^{*}\right)$ is optimal for (DII) and the two objectives have the same optimal values.

Proof. If $x^{*}$ is an optimal solution for $(\mathrm{P})$, then by Theorem 2.1 , there exist $\bar{v}, \bar{w} \in R^{n}$, and $\bar{\mu} \in R_{+}^{p}$ to satisfy the expression (2), that is, the expression (32) by substituting

$$
k_{\circ}=\frac{f\left(\bar{x}, \bar{y}_{i}^{*}\right)+\left(\bar{x}^{t} B \bar{x}\right)^{1 / 2}}{h\left(\bar{x}, \bar{y}_{i}^{*}\right)-\left(\bar{x}^{t} D \bar{x}\right)^{1 / 2}}
$$

in (2). It follows that there are $\left(\bar{s}, \bar{t}, \bar{y}^{*}\right) \in K\left(x^{*}\right)$ and $\left(x^{*}, \bar{\mu}, \bar{v}, \bar{w}\right) \in H_{2}\left(\bar{s}, \bar{t}, \bar{y}^{*}\right)$ such that $\left(x^{*}, \bar{\mu}, \bar{v}, \bar{w}, \bar{s}, \bar{t}, \bar{y}^{*}\right)$ is feasible for (DII) and such that problems (P) and (DII) have the same objective values. The optimality of this feasible solution for (DII) thus follows from Theorem 5.2.

Theorem 5.4. Strict Converse Duality. Let $x^{*}$ and $\left(\bar{z}, \bar{\mu}, \bar{v}, \bar{w}, \bar{s}, \bar{t}, \bar{y}^{*}\right)$ be the optimal solutions for (P) and (DII), respectively. Suppose that $f\left(., \bar{y}_{i}^{*}\right)+$ $(.)^{t} B \bar{w}$ and $-h\left(., \bar{y}_{i}^{*}\right)+(.)^{t} D \bar{v}$, for $i=1,2, \ldots, \bar{s}$, are respectively $\left(F, \alpha, \rho_{i}, d_{i}\right)-$ convex and $\left(F, \alpha, \bar{\rho}_{i}, \bar{d}_{i}\right)$-convex at $\bar{z}$ for all $\left(\bar{s}, \bar{t}, \bar{y}^{*}\right) \in K\left(x^{*}\right)$ and $(\bar{z}, \bar{\mu}, \bar{v}, \bar{w}) \in$ $H_{2}\left(\bar{s}, \bar{t}, \bar{y}^{*}\right)$; let $g_{j}($.$) , for j=1,2, \ldots, p$, be $\left(F, \beta_{j}, v_{j}, c_{j}\right)$-convex at $\bar{z}$, let the inequality

$$
\begin{aligned}
& \sum_{i=1}^{\bar{s}} \bar{t}_{i}\left\{\frac{\left(h\left(\bar{z}, \bar{y}_{i}^{*}\right)-\left(\bar{z}^{t} D \bar{z}\right)^{1 / 2}\right) \rho_{i} d_{i}^{2}\left(x^{*}, \bar{z}\right)}{\alpha\left(x^{*}, \bar{z}\right)}+\frac{\left(f\left(\bar{z}, \bar{y}_{i}^{*}\right)+\left(\bar{z}^{t} B \bar{z}\right)^{1 / 2}\right) \bar{\rho}_{i} \bar{d}_{i}^{2}\left(x^{*}, \bar{z}\right)}{\alpha\left(x^{*}, \bar{z}\right)}\right\} \\
& +\sum_{j=1}^{p} \bar{\mu}_{j} v_{j} \frac{c_{j}^{2}\left(x^{*}, \bar{z}\right)}{\beta_{j}\left(x^{*}, \bar{z}\right)}>0
\end{aligned}
$$

hold, and let $\nabla g_{j}\left(x^{*}\right), j \in J\left(x^{*}\right)$ be linearly independent. Then, $x^{*}=\bar{z}$; that is, $\bar{z}$ is optimal for $(\mathrm{P})$ and

$$
\sup _{y \in Y} \frac{f\left(\bar{z}, \bar{y}^{*}\right)+\left(\bar{z}^{t} B \bar{z}\right)^{1 / 2}}{h\left(\bar{z}, \bar{y}^{*}\right)-\left(\bar{z}^{t} D \bar{z}\right)^{1 / 2}}=F(\bar{z}) .
$$

Proof. We shall assume that $x^{*} \neq \bar{z}$ and reach a contradiction. From conditions similar to the proof of Theorem 5.2, we have

$$
\begin{aligned}
& \frac{\phi_{2}\left(x^{*}\right)-\phi_{2}(\bar{z})}{\alpha\left(x^{*}, \bar{z}\right)} \geq F\left(x^{*}, \bar{z} ; \sum_{i=1}^{\bar{s}} \bar{t}_{i}\left\{\left(h\left(\bar{z}, \bar{y}_{i}^{*}\right)-\left(\bar{z}^{t} D \bar{z}\right)^{1 / 2}\right)\left(\nabla f\left(\bar{z}, \bar{y}_{i}^{*}\right)+B \bar{w}\right)\right.\right. \\
&\left.\left.-\left(f\left(\bar{z}, \bar{y}_{i}^{*}\right)+\left(\bar{z}^{t} B \bar{z}\right)^{1 / 2}\right)\left(\nabla h\left(\bar{z}, \bar{y}_{i}^{*}\right)-D \bar{v}\right)\right\}\right) \\
&+\sum_{i=1}^{\bar{s}} \bar{t}_{i}\left\{\frac{\left(h\left(\bar{z}, \bar{y}_{i}^{*}\right)-\left(\bar{z}^{t} D \bar{z}\right)^{1 / 2}\right) \rho_{i} d_{i}^{2}\left(x^{*}, \bar{z}\right)}{\alpha\left(x^{*}, \bar{z}\right)}+\frac{\left(f\left(\bar{z}, \bar{y}_{i}^{*}\right)+\left(\bar{z}^{t} B \bar{z}\right)^{1 / 2}\right) \bar{\rho}_{i} \bar{d}_{i}^{2}\left(x^{*}, \bar{z}\right)}{\alpha\left(x^{*}, \bar{z}\right)}\right\},
\end{aligned}
$$




$$
\sum_{j=1}^{p} \bar{\mu}_{j} \frac{g_{j}\left(x^{*}\right)-g_{j}(\bar{z})}{\beta_{j}\left(x^{*}, \bar{z}\right)} \geq F\left(x^{*}, \bar{z} ; \sum_{j=1}^{p} \bar{\mu}_{j} \nabla g_{j}(\bar{z})\right)+\sum_{j=1}^{p} \bar{\mu}_{j} v_{j} \frac{c_{j}^{2}\left(x^{*}, \bar{z}\right)}{\beta_{j}\left(x^{*}, \bar{z}\right)} .
$$

By the feasibility of $x^{*}, \beta\left(x^{*}, \bar{z}\right)>0$, and (33), inequality (45) gives

$$
F\left(x^{*}, \bar{z} ; \sum_{j=1}^{p} \bar{\mu}_{j} \nabla g_{j}(\bar{z})\right) \leq-\sum_{j=1}^{p} \bar{\mu}_{j} v_{j} \frac{c_{j}^{2}\left(x^{*}, \bar{z}\right)}{\beta_{j}\left(x^{*}, \bar{z}\right)} .
$$

From (32), (43), and (46), along with the sublinearity of $F$, we obtain

$$
\begin{aligned}
& F\left(x^{*}, \bar{z} ; \sum_{i=1}^{\bar{s}} \bar{t}_{i}\left\{\left(h\left(\bar{z}, \bar{y}_{i}^{*}\right)-\left(\bar{z}^{t} D \bar{z}\right)^{1 / 2}\right)\left(\nabla f\left(\bar{z}, \bar{y}_{i}^{*}\right)+B \bar{w}\right)\right.\right. \\
& \left.\left.-\left(f\left(\bar{z}, \bar{y}_{i}^{*}\right)+\left(\bar{z}^{t} B \bar{z}\right)^{1 / 2}\right)\left(\nabla h\left(\bar{z}, \bar{y}_{i}^{*}\right)-D \bar{v}\right)\right\}\right) \\
& \geq-F\left(x^{*}, \bar{z} ; \sum_{i=1}^{p} \bar{\mu}_{j} \nabla g_{j}(\bar{z})\right) \\
& \geq \sum_{j=1}^{p} \bar{\mu}_{j} v_{j} \frac{c_{j}^{2}\left(x^{*}, \bar{z}\right)}{\beta_{j}\left(x^{*}, \bar{z}\right)} \\
& >-\sum_{i=1}^{\bar{s}} \bar{t}_{i}\left\{\frac{\left(h\left(\bar{z}, \bar{y}_{i}^{*}\right)-\left(\bar{z}^{t} D \bar{z}\right)^{1 / 2}\right) \rho_{i} d_{i}^{2}\left(x^{*}, \bar{z}\right)}{\alpha\left(x^{*}, \bar{z}\right)}\right. \\
& \left.+\frac{\left(f\left(\bar{z}, \bar{y}_{i}^{*}\right)+\left(\bar{z}^{t} B \bar{z}\right)^{1 / 2}\right) \bar{\rho}_{i} \bar{d}_{i}^{2}\left(x^{*}, \bar{z}\right)}{\alpha\left(x^{*}, \bar{z}\right)}\right\} .
\end{aligned}
$$

Using $\alpha\left(x^{*}, \bar{z}\right)>0$ and (47), inequality (44) yields

$$
\phi_{2}\left(x^{*}\right)-\phi_{2}(\bar{z})>0
$$

that is,

$$
\begin{aligned}
\sum_{i=1}^{\bar{s}} \bar{t}_{i}\left\{\left(h\left(\bar{z}, \bar{y}_{i}^{*}\right)-\bar{z}^{t} D \bar{v}\right)\left(f\left(x^{*}, \bar{y}_{i}^{*}\right)+x^{* t} B \bar{w}\right)\right. \\
\left.\quad-\left(f\left(\bar{z}, \bar{y}_{i}^{*}\right)+\bar{z}^{t} B \bar{w}\right)\left(h\left(x^{*}, \bar{y}_{i}^{*}\right)-x^{* t} D \bar{v}\right)\right\} \\
>\sum_{i=1}^{\bar{s}} \bar{t}_{i}\left\{\left(h\left(\bar{z}, \bar{y}_{i}^{*}\right)-\bar{z}^{t} D \bar{v}\right)\left(f\left(\bar{z}, \bar{y}_{i}^{*}\right)+\bar{z}^{t} B \bar{w}\right)\right. \\
\left.-\left(f\left(\bar{z}, \bar{y}_{i}^{*}\right)+\bar{z}^{t} B \bar{w}\right)\left(h\left(\bar{z}, \bar{y}_{i}^{*}\right)-\bar{z}^{t} D \bar{v}\right)\right\} \geq 0 .
\end{aligned}
$$


Therefore, there exists a certain $i_{\circ}$ such that

$$
\begin{aligned}
& \sum_{i=1}^{\bar{s}} \bar{t}_{i}\left\{\left(h\left(\bar{z}, \bar{y}_{i_{\circ}}^{*}\right)-\bar{z}^{t} D \bar{v}\right)\left(f\left(x^{*}, \bar{y}_{i_{\circ}}^{*}\right)+x^{* t} B \bar{w}\right)\right. \\
& \left.\quad-\left(f\left(\bar{z}, \bar{y}_{i_{\circ}}^{*}\right)+\bar{z}^{t} B \bar{w}\right)\left(h\left(x^{*}, \bar{y}_{i_{\circ}}^{*}\right)-x^{* t} D \bar{v}\right)\right\}>0 .
\end{aligned}
$$

From the above inequality and (35), it follows that

$$
\sup _{y \in Y} \frac{f\left(x^{*}, \bar{y}^{*}\right)+\left(x^{* t} B x^{*}\right)^{1 / 2}}{h\left(x^{*}, \bar{y}^{*}\right)-\left(x^{* t} D x^{*}\right)^{1 / 2}} \geq \frac{f\left(x^{*}, \bar{y}_{i_{\circ}}^{*}\right)+\left(x^{* t} B x^{*}\right)^{1 / 2}}{h\left(x^{*}, \bar{y}_{i_{\circ}}^{*}\right)-\left(x^{* t} D x^{*}\right)^{1 / 2}}>F(\bar{z}) .
$$

By strong duality theorem, we know that

$$
\sup _{y \in Y} \frac{f\left(x^{*}, \bar{y}^{*}\right)+\left(x^{* t} B x^{*}\right)^{1 / 2}}{h\left(x^{*}, \bar{y}^{*}\right)-\left(x^{* t} D x^{*}\right)^{1 / 2}}=F(\bar{z}) .
$$

Inequality (48) contradicts (49); hence, the proof is complete.

Remark 5.1. If we replace $F(x, \bar{x} ; \alpha(x, \bar{x}) \nabla f(\bar{x}))$ by $(x-\bar{x})^{t} \nabla f(\bar{x})$, for $x, \bar{x} \in X$, in Theorems 5.2-5.4, we get Theorems 1-3 in Ref. 14. Also, when $B=C=0$ in the objective function, Theorems 4.5-4.7 of Ref. 12 can be obtained; in addition, if $\alpha(x, \bar{x})=1$, for all $x, \bar{x} \in X$, Theorems $4.5-4.7$ of Ref. 8 can be found.

\section{References}

1. Schmitendorf, W. E., Necessary Conditions and Sufficient Conditions for Static Minimax Problems, Journal of Mathematical Analysis and Applications, Vol. 57, pp. 683-693, 1997.

2. TANimoto, S., Duality for a Class of Nondifferentiable Mathematical Programming Problems, Journal of Mathematical Analysis and Applications, Vol. 79, pp. 283-294, 1981.

3. YAdAV, S. R., and MukHerJeE, R. N., Duality for Fractional Minimax Programming Problems, Journal of the Australian Mathematical Society, Vol. 31B, pp. 484-492, 1990.

4. Chandra, S., and Kumar, V., Duality in Fractional Minimax Programming, Journal of the Australian Mathematical Society, Vol. 58A, pp. 376-386, 1995.

5. Bector, C. R., and Bhatia, B. L., Sufficient Optimality and Duality for a Minimax Problem, Utilitas Mathematica, Vol. 27, pp. 229-247, 1985.

6. WeIR, T., Pseudoconvex Minimax Programming, Utilitas Mathematica, Vol. 42, pp. 234-240, 1992.

7. Zalmai, G. J., Optimality Criteria and Duality for a Class of Minimax Programming Problems with Generalized Invexity Conditions, Utilitas Mathematica, Vol. 32, pp. 35-57, 1987. 
8. LIU, J. C., and Wu, C. S., On Minimax Fractional Optimality Conditions with $(F, \rho)-$ Convexity, Journal of Mathematical Analysis and Applications, Vol. 219, pp. 36-51, 1998.

9. Ahmad, I., Optimality Conditions and Duality in Fractional Minimax Programming Involving Generalized $\rho$-Invexity, International Journal of Management and Systems, Vol. 19, pp. 165-180, 2003.

10. Liang, Z. A., HuAng, H. X., and Pardalos, P. M., Optimality Conditions and Duality for a Class of Nonlinear Fractional Programming Problems, Journal of Optimization Theory and Applications, Vol. 110, pp. 611-619, 2001.

11. Liang, Z. A., HuAng, H. X., and Pardalos, P. M., Efficiency Conditions and Duality for a Class of Multiobjective Programming Problems, Journal of Global Optimization, Vol. 27, pp. 1-25, 2003.

12. LiAng, Z. A., and SHI, Z. W., Optimality Conditions and Duality for Minimax Fractional Programming with Generalized Convexity, Journal of Mathematical Analysis and Applications, Vol. 277, pp. 474-488, 2003.

13. LAI, H. C., LiU, J. C., and TANAKA, K., Necessary and Sufficient Conditions for Minimax Fractional Programming, Journal of Mathematical Analysis and Applications, Vol. 230, pp. 311-328, 1999.

14. LAI, H. C., and LEE, J. C., On Duality Theorems for Nondifferentiable Minimax Fractional Programming, Journal of Computational and Applied Mathematics, Vol. 146, pp. 115-126, 2002.

15. Mishra, S. K., WANG, S. Y., LAI, K. K., and SHI, J. M., Nondifferentiable Minimax Fractional Programming under Generalized Univexity, Journal of Computational and Applied Mathematics, Vol. 158, pp. 379-395, 2003.

16. PREDA, V., On Efficiency and Duality for Multiobjective Programs, Journal of Mathematical Analysis and Applications, Vol. 166, pp. 365-377, 1992.

17. JeYAKUMAR, V., Strong and Weak Invexity in Mathematical Programming, Mathematics of Operations Research, Vol. 55, pp. 109-125, 1985.

18. HANSON, M. A., On Sufficiency of the Kuhn-Tucker Conditions, Journal of Mathematical Analysis and Applications, Vol. 80, pp. 544-550, 1981. 